\title{
A COST-EFFECTIVE METHOD FOR CRACK DETECTION AND MEASUREMENT ON CONCRETE SURFACE
}

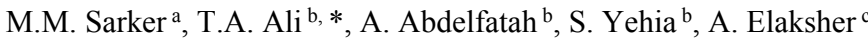 \\ a School of Civil Engineering, The University of Sydney, Sydney, Australia - msar8002@uni.sydney.edu.au \\ ${ }^{\mathrm{b}}$ Department of Civil Engineering, College of Engineering, American University of Sharjah, United Arab Emirates \\ (atarig, akmal, syehia)@aus.edu \\ ${ }^{\mathrm{c}}$ Civil Engineering Department, College of Engineering, Cal Poly Pomona University, USA afelaksher@cpp.edu
}

\section{Commission II}

KEY WORDS: crack detection, depth camera, image processing, stereovision

\begin{abstract}
:
Crack detection and measurement in the surface of concrete structures is currently carried out manually or through Non-Destructive Testing (NDT) such as imaging or scanning. The recent developments in depth (stereo) cameras have presented an opportunity for cost-effective, reliable crack detection and measurement. This study aimed at evaluating the feasibility of the new inexpensive depth camera (ZED) for crack detection and measurement. This depth camera with its lightweight and portable nature produces a 3D data file of the imaged surface. The ZED camera was utilized to image a concrete surface and the 3D file was processed to detect and analyse cracks. This article describes the outcome of the experiment carried out with the ZED camera as well as the processing tools used for crack detection and analysis. Crack properties that were also of interest were length, orientation, and width. The use of the ZED camera allowed for distinction between surface and concrete cracks. The ZED high-resolution capability and point cloud capture technology helped in generating a dense 3D data in low-lighting conditions. The results showed the ability of the ZED camera to capture the crack depth changes between surface (render) cracks, and crack that form in the concrete itself.
\end{abstract}

\section{INTRODUCTION AND BACKGROUND}

The detection and measurement of cracks on concrete structures has been of significant interest in recent years. Crack formations, widths and propagation are important indicators of the structural integrity and health of a concrete element. Methods of automatic crack detection were done conventionally through image processing (Kawamura and Miyamoto, 2003; Sato et al., 2003; Abdel-Qader et al., 2003).

Since then, seminal research work has been carried out through a percolation model which is based on the relationship with neighbouring pixels (Yamaguchi and Hashimoto, 2006). Further to this, Yamaguchi and Hashimoto (2009) proposed an improved image processing method for determining the width of cracks on concrete surfaces. In their method, the width is measured by the brightness of the crack area. The sub-order pixel accuracy of the method is evaluated and the effectiveness is validated for real world applications.

Recently, stereo cameras have become involved in crack detection and monitoring - in particular the 3D representation of the crack propagation and geometrical formation. Nakagawa et al. (2013) devised a temporal 3D data registration method based on an iterative closest point (ICP) algorithm. Similarly, Tsukuda et al. (2013) proposed a 3D method of measurement with a compensation method that incorporated equivalent strain-measurements through experiments involving four-point prism bending tests on reinforced concrete beams.
Zheng (2014) conducted one of the more seminal works of research into crack detection and measurements through an image-based reconstruction process. A crack detection algorithm was performed on point cloud meshes that were generated from images. The crack measurements from the numerical analysis were correlated with manual measurements of cracks on the test specimens and evaluated for accuracy.

The use of as-built building models and 3D mapping technology is widely acknowledged and researched in the architecture, engineering and construction (AEC) community. Although, in the past, novel methods of acquiring building model data has been identified to be labour-intensive, strenuous and time-consuming (Tang et al., 2010). Traditionally, methods of acquiring data have been done through the use of terrestrial laser scanners and photogrammetric methods. Facilitating a more efficient method of creating as-built models has led researchers to automating the process, which has been proven effective. Photogrammetric methods, such as modelling from images and video, are more portable and convenient when compared to laser scanners. Pollefeys et al. (2011) developed a processing pipeline that constructed a 3D model from hand-held cameras. This form of structure from motion recovery method has been utilised previously in research (Snavely et al., 2006; Barazetti et al., 2010; Remondino and El-Hakim, 2014). The drawback however involves the extraction of poorly textured building elements and multiple interior partitions which cause issues in the registration of visual data. Furthermore, the methods are highly dependent on pre-processing and manually encoding attributes for recognition thereby restricting scenarios

* Corresponding author 
for formal applications and lack of testing in highly cluttered areas, such as construction sites.

Recently, stereo vision systems have been implemented to recognise simple building elements, such as beams and columns. Colour and 3D data acquired from stereo vision cameras have demonstrated the potential for detecting and evaluating the nature of structural elements in buildings, especially in high noise and cluttered environments (Son and Kim, 2010; Zhu, 2011; Kim et al., 2016).

Building elements recognition is usually characterised in the following sequence of steps:

\begin{tabular}{|c|c|}
\hline 1. & Remote sensing \\
\hline 2. & 3D point cloud \\
\hline 3. & Element recognition \\
\hline & a. Type \\
\hline & $\begin{array}{l}\text { b. Boundaries } \\
\text { c. Attributes }\end{array}$ \\
\hline
\end{tabular}

The recognition of building elements comes as a result of spatial and visual data. In the case of spatial data - methods such as laser scanners, 3D depth cameras and photogrammetry is typically utilised. In this case, data is derived from built in knowledge of the element's attributes: semantic information of the building elements (walls, floors, ceilings, columns etc.), geometrical and spatial relationships between the elements, and object boundaries and constraints (Nuechter et al., 2003; Pu and Vosselman, 2009; Huber et al., 2011). Since Building Information Modelling (BIM) cannot simply be carried out through the interpolation of point-clouds in a mesh, there is a need for additional information such materiality, energy usage and construction stages for parametric models. This is however outside the scope of this study.

Previous methods of acquiring data for generating as-built models have been through detailed information registered by laser scanners and photogrammetry. Despite the high level of accuracy that is generally achieved, previous research has indicated that these methods are labour intensive. Adan et al. (2011) conducted experiments in which rich semantic information was retrieved from 225 locations in 40 rooms resulting in over 3 billion 3D measurements. The process was highly labour-intensive and prone to errors in measurement and subsequent post-processing.

There has been promising results from the use of depth cameras for mapping indoor building environment. Henry et al. (2014) integrated the use of visual and shape information available from RBG-D cameras in order to map dense indoor environments. The mapping system utilises a joint optimisation algorithm which assists in aligning shape and visual features. The novel approach also allows for the detection of loops closures by matching data frames to previously collected frames. The end result involved pose optimisation to create globally consistent maps within a complicated indoor context. Zhu and Donia (2013) further investigated the accuracy and automotive processes in modelling as-built building environments through several pilot studies. The automatic creation of $3 \mathrm{D}$ as-built models is a challenging process due to several constraints. The lack of adequate lighting and texture quality contributes to shortcomings in visual data, whilst the large number of partitions and elements within a space contribute to lack of spatial data properly registered. The studies found that RGB-D cameras, in the case of the study a Kinect was used, was accurate to the centimetre, when compared to sensing data retrieved from a Total Station. The fusion of spatial and visual data proved to show promise in the ability to recognise and model building elements within an indoor environment. Chen et al. (2016) carried out experiments in order to validate the use of low-cost RGB-D depth cameras for indoor data collection. In their paper, the proposed method involved the use of a sensor array through use of multiple Kinect sensors and a prototype indoor scanner. Chen et al. (2016) proposed a novel framework for utilising consumer depth cameras in mapping indoor environments. The requirements for indoor RGB data collection typically rely on low-cost and automated processes - through wider viewing angles and efficient retrieval processes. The use of ZED stereo cameras allows for a separate avenue of retrieving spatial and visual data to generate as-built models for use in BIM.

Despite the number of present research looking into low-cost building modelling, challenges still arise in creating highquality semantic models in low-light and texture-poor building elements. Several commercial RBG-D cameras utilised in previous research have shown promise, and have been well documented, however, issues still arise in post-processing. Namely, the conversion of point-cloud data to semantic 3D models with high-quality textures and renders are still being investigated. Furthermore, conversion of point-cloud data into $x y z$ models for use in rendering software such as AutoCAD still needs to be addressed.

\section{ZED STEREO CAMERA}

The ZED stereo camera (Figure 1) employs dual 4MP cameras that can capture $2 \mathrm{~K} 3 \mathrm{D}$ video, able to operate in low-light conditions and challenging high noise environments (Stereolabs, 2017). When compared to depths cameras used in previous research (Henry et al., 2014; Zhu and Donia, 2013a; Zhu and Donia, 2013b; Chen et al., 2016) - this model includes a high frame-rate and a wider field of view, 16:9 aspect ratio and can capture $110^{\circ}$ wide-angle video and depth. The features include, a high-resolution and high-frame rate 3D video capture, depth perception (both indoors and outdoors) at up to 20,000 mm, 6-DOF positional tracking, and a large-scale 3D mapping using ZEDfu software (Figure 3 ) which is included in the installation package. Table 1 shows a comparison of the video modes available on the ZED camera:

\begin{tabular}{|l|l|l|}
\hline $\begin{array}{l}\text { Video } \\
\text { Mode }\end{array}$ & $\begin{array}{l}\text { Frames per } \\
\text { second }\end{array}$ & Output \\
\hline $2.2 \mathrm{~K}$ & 15 & $4416 \times 1242$ \\
\hline $1080 \mathrm{p}$ & 30 & $3840 \times 1080$ \\
\hline $720 \mathrm{p}$ & 60 & $2560 \times 720$ \\
\hline WVGA & 100 & $1344 \times 376$ \\
\hline
\end{tabular}

Table 1. Video Mode Information

In order to assess the accuracy of depth camera, Zhu and Donia (2013b) conducted experiments in which the measurements of coordinate systems registered by a Total Station and Kinetic depth camera were examines. Table 2 shows the two examples of experimental results which compare the distance values measured by the Total Station and Kinetic. From their results, 70 experimental data were retrieved and percentage errors were $3.6 \mathrm{~cm}$ and $2.9 \%$. 


\begin{tabular}{|l|l|l|}
\hline & No. 34 & No. 35 \\
\hline Distance (Total Station) (m) & 0.843 & 4.123 \\
\hline Distance (Kinetic) (m) & 0.818 & 4.166 \\
\hline Absolute Error & 0.025 & 0.043 \\
\hline Percentage Error & $2.97 \%$ & $1.04 \%$ \\
\hline
\end{tabular}

Table 2. Experimental Results of Sensing Data (Zhu and Donia, 2013a)

Current methods of registration and collection of visual and spatial date have been accomplished through the use of RGB-D cameras. Methods of progressively mapping scenes through RBG-D cameras create more detailed and accurate scene registrations and have less reliance on visual cues to create 3D scenes of a local environment. Table 3 details a comparison of specific criteria that govern the efficacy of 3D element mapping and registration.

\begin{tabular}{|l|l|l|}
\hline Criteria & $\begin{array}{l}\text { Point Cloud based } \\
\text { methods }\end{array}$ & $\begin{array}{l}\text { Colour based } \\
\text { methods }\end{array}$ \\
\hline $\begin{array}{l}\text { Sensing data } \\
\text { properties }\end{array}$ & $\begin{array}{l}\text { Accurate and } \\
\text { detailed }\end{array}$ & $\begin{array}{l}\text { Not accurate and } \\
\text { detailed }\end{array}$ \\
\hline Scene registration & $\begin{array}{l}\text { Point cloud } \\
\text { distance }\end{array}$ & $\begin{array}{l}\text { Visual features } \\
\text { matching }\end{array}$ \\
\hline $\begin{array}{l}\text { Building Element } \\
\text { Recognition }\end{array}$ & $\begin{array}{l}\text { One recognition } \\
\text { cue only (spatial } \\
\text { feature) }\end{array}$ & $\begin{array}{l}\text { Multiple } \\
\text { Recognition cues }\end{array}$ \\
\hline
\end{tabular}

Table 3. Comparison of point cloud and colour based methods (Zhu and Donia, 2013a)

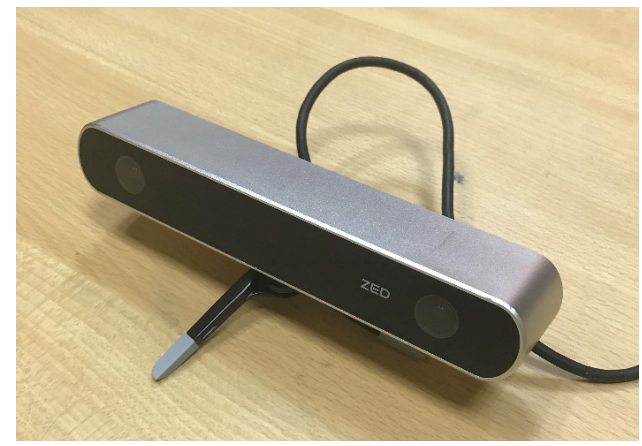

Figure 1. ZED Stereo Camera

\section{EXPERIMENT AND RESULTS}

The first process involved was registering a region with a defined crack that has propagated into the crack surface as a 3D point cloud using the ZED depth viewer (Figure 2). The initial point cloud registration was edited on MeshLab to correct the edges and create a relatively equal sided shape (Figure 4). This was done purely for aesthetic purposes for presentation rather than practicality. MATLAB was utilized for post-processing and image analysis due to the simplicity and robustness of the software. As MATLAB includes a third-party integration with $Z E D$, the use of the camera was also possible without the use of ZED's depth viewer. Following the registration (Figure 5,6), an image-based analysis algorithm was implemented to retrieve the crack segments within the point cloud (Figure 7, 8). The algorithm allowed for the distinction between paint cracks, and the cracks that formed on the concrete surface by analysing its depth from point cloud. Figure 9 illustrates the relative depths of the major cracks to the surface of the region. The retrieval of crack region's properties was conducted through MATLAB through Euclidean distance calculations and image analysis, including: the crack length and depth of major $\operatorname{crack}(\mathrm{s})$, the total surface length and area of cracking region. Table 4 summarizes the results of the analysis and illustrates that the relative accuracy of the method vs. empirical measurements is $5-9 \%$.

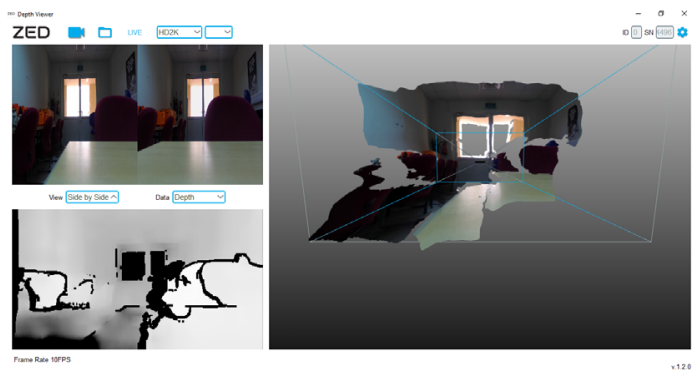

Figure 2. ZED Depth Viewer

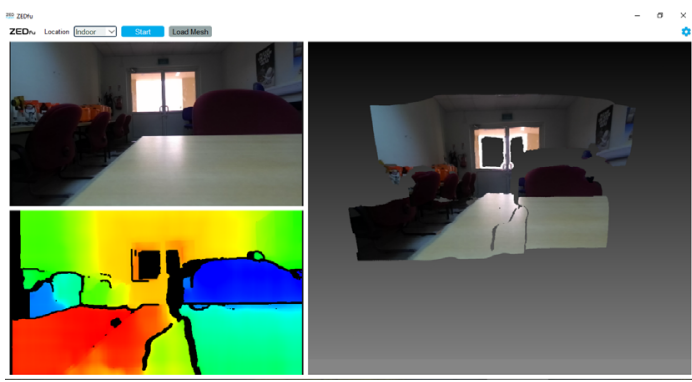

Figure 3. ZEDfu

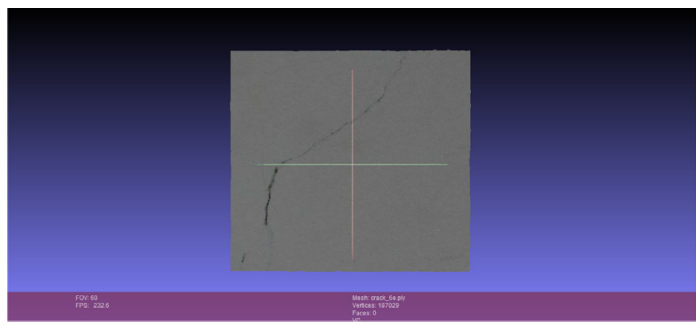

Figure 4. 3D Point Cloud cropping on MeshLab

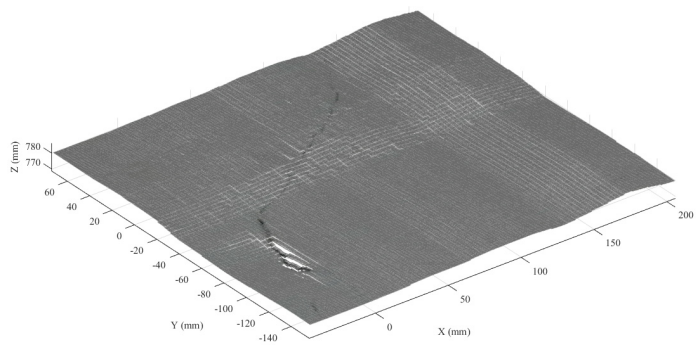

Figure 5. Registered 3D Point Cloud of Cracked Surface

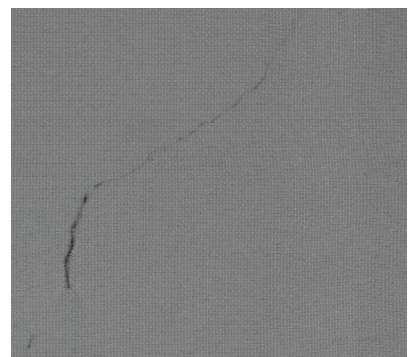

Figure 6. Point Cloud of Cracked Surface 


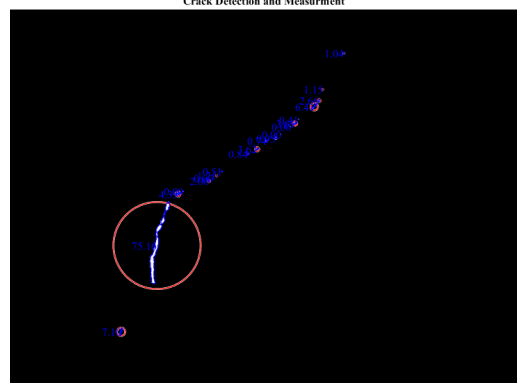

Figure 7. Crack Detection and Measurement
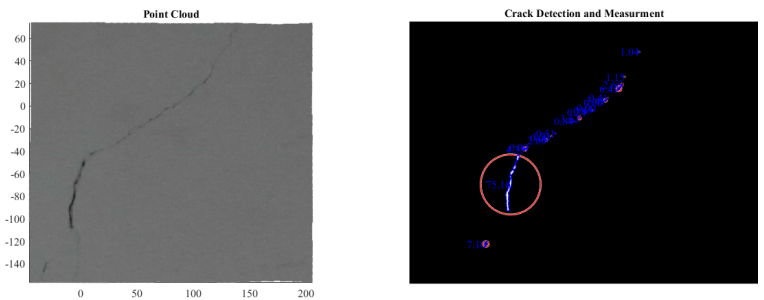

Figure 8. Comparison of registered point cloud and detection image

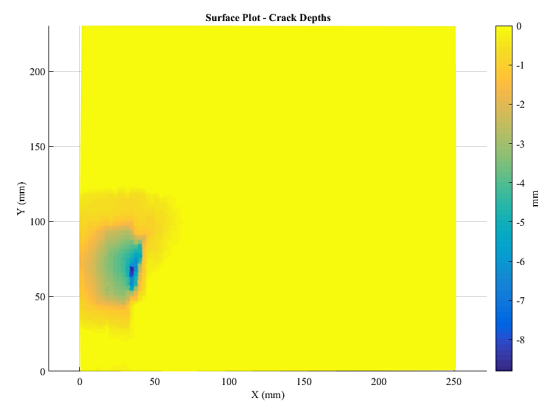

Figure 9. Relative depth of major cracks on surface

\begin{tabular}{|l|l|}
\hline Property & Measurement \\
\hline Major Crack & $75.16 \mathrm{~mm}$ \\
\hline Major Crack (Empirical) & $71 \mathrm{~mm}$ \\
\hline Percentage Error (\%) & $5.5 \%$ \\
\hline Major Crack Depth & $8.81 \mathrm{~mm}$ \\
\hline Total Surface Crack & $285.16 \mathrm{~mm}$ \\
\hline Total Surface Crack (Empirical) & $259 \mathrm{~mm}$ \\
\hline Percentage Error (\%) & $9.3 \%$ \\
\hline Total Crack Area & $1062 \mathrm{~mm}^{2}$ \\
\hline Area of Region of Interest & $57960 \mathrm{~mm}^{2}$ \\
\hline
\end{tabular}

Table 4. Results of CDM Test

\section{CONCLUDING REMARKS}

The challenge of indoor environment mapping for 3D as-built modelling of building is an important aspect of Architecture, Engineering and Construction to consider. The limit of available technology, such as cost, labour and post-processing, has made it difficult to capture the automatic generation of models. The recent development of the ZED depth camera provides a novel method for capturing high quality $3 \mathrm{D}$ environments in real-time with little manual processing involved compared to traditional methods of analysis. The feasibility studies outline methods that are used to characterise the potential of camera based on its properties and capabilities.
A pilot study involving crack detection technology and processing also showcased the potential for the technology to develop automatic capture and analysis of cracks forming on concrete structures. Whilst research into the potential of ZED depth camera is at the basic stage, experimental methods have enormous potential for accurate and robust recognition and modelling of infrastructure. Future work will focus on developing a solid framework for the development of more sophisticated and accurate modelling techniques, such as topometric localisation, real-time mapping of large environments, visual reconstruction of civil engineering project, and recognition of building and structural elements. In addition to that, results obtained by the proposed procedures will be compared to the outcomes of some other NDT techniques, such as Ground Penetrating Radar (GPR).

\section{REFERENCES}

Abdel-Qader, I., Abudayyeh, O. and Kelly, M. E., 2003. Analysis of edge-detection techniques for crack identification in bridges. Journal of Computing in Civil Engineering, 17(4), pp. 255-263.

Adan, A., Xiong, X., Akinci, B. and Huber, D., 2011. Automatic Creation of Semantically Rich 3D Building Models from Laser Scanner Data. Proceedings of the International Symposium on Automation and Robotics in Construction (ISARC), pp. 343-348.

Barazzetti, L., Scaioni, M. and Remondino, F., 2010. Orientation and 3D modelling from markerless terrestrial images: combining accuracy with automation. The Photogrammetric Record, 25(132), pp. 356-381.

Chen, C., Yang, B. and Song, S., 2016. Low Cost and Efficient 3D Indoor Mapping Using Multiple Consumer RGB-D Cameras. International Archives of the Photogrammetry, Remote Sensing \& Spatial Information Sciences, 41.

Henry, P., Krainin, M., Herbst, E., Ren, X. and Fox, D., 2012. RGB-D mapping: Using Kinect-style depth cameras for dense $3 \mathrm{D}$ modeling of indoor environments. The International Journal of Robotics Research, 31(5), pp. 647-663.

Henry, P., Krainin, M., Herbst, E., Ren, X. and Fox, D., 2014. RGB-D mapping: Using depth cameras for dense 3D modeling of indoor environments. Experimental robotics: Springer, pp. $477-491$.

Huber, D., Akinci, B., Oliver, A. A., Anil, E., Okorn, B. E. and Xiong, X., 2011. Methods for automatically modeling and representing as-built building information models. Proceedings of the NSF CMMI Research Innovation Conference.

Izadi, S., Kim, D., Hilliges, O., Molyneaux, D., Newcombe, R., Kohli, P., Shotton, J., Hodges, S., Freeman, D. and Davison, A., 2011. KinectFusion: real-time 3D reconstruction and interaction using a moving depth camera. Proceedings of the 24th annual ACM symposium on User interface software and technology: ACM, pp. 559-568.

Kawamura, K., Miyamoto, A. and Nakamura, H., 2003. Proposal of a crack pattern extraction method from digital images using an interactive genetic algorithm. Doboku Gakkai Ronbunshu, 2003(742), pp. 115-131. 
Kim, H., Campos, T. and Hilton, A., 2016. Room Layout Estimation with Object and Material Attributes Information using a Spherical Camera. Fourth International Conference on $3 D$ Vision, pp. 519-527.

Nießner, M., Zollhöfer, M., Izadi, S. and Stamminger, M., 2013. Real-time 3D reconstruction at scale using voxel hashing. ACM Transactions on Graphics (TOG), 32(6), pp. 169.

Nuechter, A., Surmann, H., Lingemann, K. and Hertzberg, J., 2003. Consistent 3D model construction with autonomous mobile robots. Annual Conference on Artificial Intelligence, Springer, pp. 550-564.

Pollefeys, M., Nistér, D., Frahm, J.-M., Akbarzadeh, A., Mordohai, P., Clipp, B., Engels, C., Gallup, D., Kim, S.-J. and Merrell, P., 2008. Detailed real-time urban 3d reconstruction from video. International Journal of Computer Vision, 78(2-3), pp. 143-167.

$\mathrm{Pu}, \quad$ S. and Vosselman, G., 2009. Knowledge based reconstruction of building models from terrestrial laser scanning data. ISPRS Journal of Photogrammetry and Remote Sensing, 64(6), pp. 575-584.

Remondino, F., Spera, M. G., Nocerino, E., Menna, F. and Nex, F., 2014. State of the art in high density image matching. The Photogrammetric Record, 29(146), pp. 144-166.

Snavely, N., Seitz, S. and Szeliski, R., 2006 Photo Tourism: Exploring image collections in 3D. www.cs.cornell.edu/ snavely/bundler.

Son, H. and Kim, C., 2010. 3D structural component recognition and modeling method using color and $3 \mathrm{D}$ data for construction progress monitoring. Automation in Construction, 19(7), pp. 844-854.

Stereolabs, 2017. https://www.stereolabs.com/. Website last accessed on July 13, 2017.
Tang, P., Huber, D., Akinci, B., Lipman, R. and Lytle, A., 2010. Automatic reconstruction of as-built building information models from laser-scanned point clouds: A review of related techniques. Automation in construction, 19(7), pp. 829-843.

Tsukuda, Y., Miyasato, Y. and Midori, M., 2013. Proposal of early detection method of bending cracks using digital image correlation method for $\mathrm{RC}$ beams in the period of progress. Materials 62 (11), pp. 702-709. (tr. Japanese)

Xiong, X., Adan, A., Akinci, B. and Huber, D., 2013. Automatic creation of semantically rich $3 \mathrm{D}$ building models from laser scanner data. Automation in Construction, 31, pp. 325-337.

Yamaguchi, T. and Hashimoto, S., 2006. Automated crack detection for concrete surface image using percolation model and edge information. 32nd Annual Conference on IEEE Industrial Electronics, IECON 2006, pp. 3355-3360.

Zheng, P., 2014. Crack Detection and Measurement Utilizing Image-Based Reconstruction. https://vtechworks.lib.vt.edu/bitstream/handle/10919/48963/cra ck_detection_and_measurement_utilizing_image_based_recons truction.pdf? sequence $=1 \&$ isAllowed $=y$, last accessed on October 20, 2017

Zhu, Z., 2011. Column recognition and defects/damage properties retrieval for rapid infrastructure assessment and rehabilitation using machine vision. Doctoral dissertation, Georgia Institute of Technology.

Zhu, Z. and Donia, S., 2013. Potentials of RGB-D Cameras in As-Built Indoor Environment Modeling. Computing in Civil Engineering (2013), pp. 605-612.

Zhu, Z. and Donia, S., 2013. Spatial and visual data fusion for capturing, retrieval, and modeling of as-built building geometry and features,. Visualization in Engineering, 1(1), pp. 10. 